Hier steht eine Anzeige.

Springer

\title{
Radiotherapieinduzierten Knochenschmerzen vorbeugen
}

\author{
Nach einer palliativen Radiotherapie zur Behandlung symptomatischer \\ Knochenmetastasen kann es zu einem vorübergehenden Aufflackern der \\ Schmerzen kommen. In einer placebokontrollierten Studie wurde getestet, \\ ob Dexamethason dies wirksam verhindert.
}

$D^{i,}$ ie vorübergehende Verschlechterung von Schmerzen nach einer palliativen Radiotherapie bei symptomatischen Knochenmetastasen tritt mit einer Inzidenz von $30-40 \%$ ein und ist für die Betroffenen sehr belastend. Vermutlich geht sie auf die Wirkung inflammatorischer Zytokine zurück, weshalb Dexamethason zur Prophylaxe oder Abschwächung geeignet sein könnte.

In der doppelt-verblindeten, randomisierten, placebokontrollierten Phase-IIIStudie wurden 298 Patienten auf entweder $4 \mathrm{mg}$ Dexamethason $(\mathrm{n}=148)$ oder Placebo $(n=150)$ oral eine Stunde vor Beginn der Radiotherapie (Einzeldosis von 8 Gy auf eine Knochenmetastase Tag 0) und in den folgenden vier Tagen einmal täglich (Tag 1 bis 4) randomisiert. Die Studienteilnehmer litten an einer oder mehreren Knochenmetastasen einer nicht hämatologischen Krebserkrankung - am häufigsten Lungenkarzinome (28\%), Prostatakarzinome (25\%) und Mammakarzinome (22\%).

Vor der Therapie und in den zehn Tagen danach wurden sie täglich zu ihren Schmerzen und dem Opioid-Konsum befragt. Zu Beginn sowie an den Tagen 10 und 42 nach der Radiotherapie füllten sie zudem den European Organisation for Research and Treatment of Cancer (EORTC) quality of life QLQ-C15-PAL, das bone metastases module (EORTC QLQ-BM22) und den Dexamethasone Symptom Questionnaire aus. Als Schmerzaufflackern galt eine Steigerung der Schmerzen auf einer Skala von 0 bis 10 um mindestens zwei Punkte ohne, dass die Schmerztherapie zurückgefahren worden wäre, oder eine $25 \%$ ige $\mathrm{Zu}$ nahme der Analgesie ohne Verbesserung der Schmerzen an den Tagen 0 bis 10, gefolgt von einem Rückgang auf das Ausgangsniveau oder darunter.

39 (26\%) Patienten der Dexamethason-Gruppe und 53 (35\%) der Placebo- gruppe erlebten in den Tagen 0 bis 10 ein Schmerzaufflackern (Differenz 8,9\%; unter $95 \%$ Konfidenzegrenze (CB) 0,0; one-sided $p=0,05)$. In den Tagen 0 bis 5 traten aufflackernde Schmerzen bei 29 (20\%) Patienten der DexamethasonGruppe und 46 (31\%) Patienten der Placebogruppe auf (Differenz 11,1\%; niedrige $95 \%$ CB 2,8; one side $\mathrm{p}=0,03$ ).

Zudem waren die Schmerzen bei den Patienten der Dexamethason-Gruppe am Tag 10 im Vergleich zu Tag 0 stärker zurückgegangen als in der Placebogruppe. Dies war allerdings nicht signifikant $(-2,37$ vs. $-1,85 ; p=0,09)$. Hingegen war der stärkere Rückgang der Schmerzen zwischen den Tagen 0 bis 5 in der Dexamethason-Gruppe im Vergleich zu Placebo signifikant $(-1,79$ vs. $-1,09 ; \mathrm{p}=0,01)$.

Am Tag 10 hatten die Patienten der Dexamethason-Gruppe signifikant weniger Übelkeit und seltener funktionale Beeinträchtigungen als zu Beginn der Studie. Auch im Vergleich zur Placebogruppe schnitten sie in diesen Punkten besser ab, zudem war ihr Appetit besser.

Fazit: Im Vergleich zu Placebo reduziert Dexamethason bei der Bestrahlung von Knochenmetastasen das Auftreten radiotherapieinduzierter Schmerzen. Des Weiteren verbessern sich Übelkeit, der Appetit und funktionale Beeinträchtigungen ohne, dass schwerwiegende $\mathrm{Ne}$ benwirkungen zu befürchten wären.

Kathrin von Kieseritzky

Chow $\mathrm{E}$ et al. Dexamethasone in the prophylaxis of radiation-induced pain $f$ are after palliative radiotherapy for bone metastases: a double-blind, randomised placebo-controlled, phase 3 trial. Lancet Oncol. 2015; 16 (15): $1463-72$ 